\title{
Evaluación positiva de medicamentos: marzo, abril y mayo 2014
}

\author{
Tabakov A. ${ }^{1}$, García Luque A. ${ }^{2}$, Sanchez López P. ${ }^{3}$, Cabanes Mariscal MA. ${ }^{4}$ \\ Sanid. mil. 2014; 70 (3): 191-196; ISSN: 1887-8571
}

\section{RESUMEN}

Se reseñan los medicamentos ya evaluados por la Agencia Española de Medicamentos y Productos Sanitarios hechos públicos en abril, mayo y junio de 2014, y considerados de mayor interés para el profesional sanitario. Se trata de opiniones técnicas positivas que son previas a la autorización y puesta en el mercado del medicamento.

PALABRAS CLAVE: Acido Folico, Neocepri ${ }^{\circledR}$, Ataluren, Translarna ${ }^{\circledR}$, Brinzolamida, Brimonidina Tartrato, Simbrinza ${ }^{\circledR}$, Empagliflozina, Jardiance ${ }^{\circledR}$, Etarfolatida, Folcepri ${ }^{\circledR}$, Obinutuzumab, Gazyvaro ${ }^{\circledR}$, Peginterferon Beta- ${ }^{\text {a }}$, Plegridy ${ }^{\circledR}$, Siltuximab, Sylvant ${ }^{\circledR}$, Simeprevir, Olysio ${ }^{\circledR}$, Simoctocog Alfa, Nuwiq ${ }^{\circledR}$, Tacrolimus, Envarsus ${ }^{\circledR}$, Trametinib, Mekinist ${ }^{\circledR}$, Vedolizumab, Entyvio ${ }^{\circledR}$, Vintafolida, Vynfinit ${ }^{\circledR}$.

\section{Positive assessment of drugs: March, April and May 2014}

SUMMARY: The drugs assessed by the Spanish Agency for Medicines and Health Products made public in April, May and June of 2014, and considered of interest to the healthcare professional, are reviewed. These are positive technical reports prior to the authorization and placing on the market of the product.

KEY WORDS: Acido Folico, Neocepri ${ }^{\circledR}$, Ataluren, Translarna ${ }^{\circledR}$, Brinzolamida, Brimonidina Tartrato, Simbrinza ${ }^{\circledR}$, Empagliflozina, Jardiance $^{\circledR}$, Etarfolatida, Folcepri ${ }^{\circledR}$, Obinutuzumab, Gazyvaro ${ }^{\circledR}$, Peginterferon Beta-1 ${ }^{\text {a }}$, Plegridy ${ }^{\circledR}$, Siltuximab, Sylvant ${ }^{\circledR}$, Simeprevir, Olysio $^{\circledR}$, Simoctocog Alfa, Nuwiq ${ }^{\circledR}$, Tacrolimus, Envarsus ${ }^{\circledR}$, Trametinib, Mekinist ${ }^{\circledR}$, Vedolizumab, Entyvio ${ }^{\circledR}$, Vintafolida, Vynfinit ${ }^{\circledR}$.

\section{ACIDO FOLICO (NEOCEPRI $\left.{ }^{\circledR}\right)^{1,2}$}

Se trata de ácido fólico usado como agente radiodiagnóstico para cáncer de ovario. Se administra por vía intravenosa antes de realizar imagen mediante tomografía computarizada por emisión de fotón único (SPECT) con 99mTc-etarfolatida. Uniéndose al receptor de folato, mejora la calidad de la imagen obtenida.

Junto con etarfolida, el Comité de la Agencia Europea de Medicamentos sobre Medicamentos de Uso Humano (CHMP) ha recomendado de manera condicional la autorización de comercialización de Neocepri $^{\circledR}$, quedando pendiente el titular de la autorización de comercialización de proporcionar más datos clínicos posteriormente. En los ensayos clínicos descritos en el apartado de etarfolida, Neocepri ${ }^{\circledR}$ ha mostrado que disminuye la actividad de fondo observada por imagen SPECT en tejidos no diana, incluyendo los intestinos, hígado, riñón y bazo, aumentando por lo tanto la calidad de la imagen.

Fue designado como medicamento huérfano el 10 de septiembre de 2012. El CHMP ha concedido la autorización de su comercialización en marzo de 2014.

\footnotetext{
1 MIR.

2 Cte. Médico. Servicio de Farmacología Clínica. Hospital Central de la Defensa Gómez Ulla. Madrid. España.

${ }^{3}$ Cte. Farmacéutico.

${ }^{4}$ Tcol. Farmacéutico. Servicio de Farmacia Hospitalaria. Hospital Central de la Defensa Gómez Ulla. Madrid. España.
}

Dirección para correspondencia: aleksandratdm@gmail.com

Recibido: 20 de junio de 2014

Aceptado: 2 de julio de 2014

\section{ATALUREN (TRANSLARNA $\left.{ }^{\circledR}\right)^{3,4}$}

Se trata de un principio activo con el mecanismo de acción desconocido que permite que la mutación del gen de la distrofina se omita durante la síntesis de esta proteína, produciéndose la proteína completamente funcional. Se trata de una mutación sin sentido que resulta en una distrofina acortada que no funciona correctamente, lo que produce síntomas característicos para la distrofia muscular de Duchenne (DMD). Antes del inicio del tratamiento, la presencia de esta mutación debe ser determinada mediante pruebas genéticas.

Está indicado en el tratamiento de la DMD causada por la mutación del gen de la distrofina, en pacientes ambulantes a partir de 5 años. La autorización se basa en un ensayo pivotal fase II, en el que ataluren ha retrasado el deterioro de la marcha en un subgrupo de pacientes con DMD mayores de 5 años. Las reacciones adversas (RAM) más comunes fueron dolor de cabeza, náuseas y vómitos.

Fue designado como medicamento huérfano el 27 Mayo del 2005. El CHMP ha recomendado su autorización condicional, siendo pendientes datos completos sobre la eficacia, procedentes de un estudio actualmente en marcha.

\section{BRINZOLAMIDA / BRIMONIDINA TARTRATO $\left(S I M B R I N Z A^{\circledR}\right)^{3,5,6}$}

Simbrinza ${ }^{\circledR}$ contiene dos principio activos: brinzolamida y brimonidina tartrato. La brinzolamida disminuye la producción de humor acuoso inhibiendo la anhidrasa carbónica en el epi- 
telio ciliar, lo que lleva a la reducción de la presión intraocular (PIO). La brimonidina actúa como agonista sobre el receptor alfa2-adrenérgico en el epitelio ciliar suprimiendo la formación de humor acuoso.

Está indicada en reducción de la PIO elevada en pacientes adultos con glaucoma de ángulo abierto o hipertensión ocular en los que la monoterapia consigue una reducción insuficiente de la PIO. Se presenta en forma de suspensión de 1\%/0.2\%. Dosis recomendada es de una gota en el ojo afectado tres veces al día. Dos ensayos clínicos de 3 meses de duración se han realizado para comparar la eficacia de este fármaco compuesto frente al tratamiento con cada uno de los principios activos por separado. La diferencia en la reducción de la PIO se ha mostrado favorable y estadísticamente significativa para $\operatorname{Simbrinz} a^{\circledR}$ en ambos estudios. Las RAM más comunes fueron hiperemia ocular, molestia ocular, visión borrosa y alergia ocular.

El CHMP ha concedido la autorización de comercialización a Simbrinza $a^{\circledR}$ el 22 de mayo de 2014.

\section{EMPAGLIFLOZINA (JARDIANCE $\left.{ }^{\circledR}\right)^{1,7-9}$}

Empagliflozina actua como inhibidor competitivo reversible y altamente selectivo del cotransportador de sodio y glucosa 2 (SGLT2) en el riñón, aumentando de esa manera la excreción de glucosa en la orina y por tanto disminuyendo la glucemia de los pacientes con diabetes tipo 2 (DM2).

Esta indicado como monoterapia en adultos con DM2 en quienes el uso de metformina se considera inadecuado por intolerancia, cuando la dieta y el ejercicio no logran un control suficiente de la glucemia. También está indicado para uso combinado con otros medicamentos hipoglucemiantes como la insulina. Se presenta en forma de comprimido recubierto con película, de $10 \mathrm{mg}$ y de $25 \mathrm{mg}$ y se toma una vez al día. Debido a su mecanismo de acción, el tratamiento con empagliflozina no debe iniciarse en pacientes con una TFGe $<60 \mathrm{ml} / \mathrm{min} / 1,73 \mathrm{~m}^{2}$ o un $\mathrm{CrCl}<60 \mathrm{ml} / \mathrm{min}$.

La eficacia de empagliflozina, definida como el cambio significativo en la HbAlc desde el nivel basal en comparación con placebo, a dosis de 10 y de $25 \mathrm{mg}$ administrado una vez al día, ha sido demostrada en el ensayo clínico con 14.500 pacientes. Este ensayo ha sido compuesto por cuatro estudios que han consistido en la evaluación de las dos dosis de empagliflozina frente a placebo: como monoterapia en pacientes con función renal conservada y en pacientes con insuficiencia renal, como terapia adyuvante de metformina y metformina más sulfonilurea y como terapia adyuvante de pioglitazona y pioglitazona más metformina. Las RAM observadas con más frecuencia fueron: hipoglucemia (junto con insulina o sulfonilurea), infecciónes genitales y del tracto urinario, prurito y poliuria/ polaquiuria.

El CHMP ha concedido la autorización de comercialización a Jardiance ${ }^{\circledR}$ en marzo de 2014.

\section{ETARFOLATIDA $\left(\text { FOLCEPRI }{ }^{\circledR}\right)^{10-12}$}

Etarfolatida es un medicamento para uso diagnostico que consiste en ácido fólico y un péptido quelante de tecnecio-99m
(99mTc). Tras el marcado radioactivo, la etarfolatida se utiliza en imagen por SPECT para detectar células que expresan el receptor de folato, característico para varios tipos de cáncer, incluido el cáncer de ovario. Datos clínicos han mostrado que los pacientes con metastasis positivos para folato identificadas con etarfolatida, son los que más se benefician de tratamiento con vintafolida, nuevo citostático que se libera preferentemente en las células cancerosas que expresan este receptor.

El CHMP ha recomendado de manera condicional la autorización de comercialización de Folcepri ${ }^{\circledR}$, requiriendo datos adicionales sobre su eficacia. Fue designado como medicamento huérfano el 10 de septiembre de 2012.

Se presenta en forma de kit para preparación radiofarmaceutica, de 100 mcg. Después de radiomarcado con 99mTc, la etarfolatida está indicada para su uso en SPECT, en combinación con tomografía computarizada (TC) ó resonancia magnética (RM), para seleccionar pacientes adultas con cáncer de ovario candidatas a recibir tratamiento con vintafolida. El ácido fólico intravenoso debe administrarse antes que 99mTc-etarfolatida para mejorar la calidad de las imágenes obtenidas mediante SPECT.

La autorización se basa en los resultados de un estudio aleatorio de fase IIb llevado a cabo en pacientes con cáncer de ovario platino resistente a los que se ha tratado con vintafolida tras la administración de etarfolida y la realización de SPECT. La eficacia de vintafolida, medida como supervivencia sin progresión (progression-free survival, PFS), ha sido mayor en el grupo de pacientes cuyas lesiones han sido marcadas con ácido fólico en su totalidad (FR 100\%) frente al grupo de FR (10\% a 90\%): 15.2 frente a 7.4 semanas, respectivamente, con un PFS hazard ratio (IC 95\%) entre dos subgrupos de 0.797 $(0.362,1.756)$. Un ensayo clínico fase III, randomizado y doble ciego, que evalúa la eficacia de etarfolatida y vintafolatida en cáncer de ovario platino resistente está actualmente en proceso. La RAM observada con más frecuencia durante el desarrollo clínico fue prurito.

\section{OBINUTUZUMAB (GAZYVARO $\left.{ }^{\circledR}\right)^{3,13,14}$}

Obinutuzumab es un anticuerpo recombinante monoclonal que se une al antígeno de transmembrana CD20 en la superficie de los linfocitos pre-B y maduros, ayudando así al sistema inmunológico para matar las células cancerosas. Está indicado, en combinación con clorambucilo, para el tratamiento de pacientes adultos con la leucemia linfocítica crónica (LLC), no tratados previamente y con comorbilidades para los que el tratamiento con fludarabina a dosis plena no es adecuado.

El principal estudio en el que se basa la recomendación de Gazyvaro $^{\circledR}$ es un ensayo de fase III que ha incluido 781 pacientes con LLC no tratados previamente. El estudio mostró que los pacientes tratados con Gazyvaro $^{\circledR}$ en combinación con clorambucil vivieron significativamente más tiempo sin que la enfermedad empeorará en comparación con los pacientes tratados con clorambucil (26,7 vs 11,1 meses) o rituximab más clorambucil (26,7 vs 15,2 meses). Las reacciones relacionadas con la perfusión, neutropenia e infecciones fueron algunos de los eventos adversos más comunes reportados durante los ensayos. 
Fue designado como medicamento huérfano el 10 de octubre del 2012. En Estados Unidos fue aprobado en noviembre de 2013.

\section{PEGINTERFERON BETA-1a (PLEGRIDY $\left.{ }^{\circledR}\right)^{3,15,16}$}

Se trata de una forma de interferon beta 1-a pegilado, con actividad inmunomoduladora. Los interferones beta aumentan la respuesta antiinflamatoria y reducen la respuesta pro-inflamatoria inmunitaria. El proceso de pegilacion del principio activo extiende su vida media lo que permite alargar los intervalos de dosificación. Está indicado en pacientes adultos para el tratamiento de esclerosis múltiple remitente recidivante (EMRR), forma más común de la EM. Su mecanismo de acción exacto no se conoce.

Se presenta en forma de solución para inyección subcutánea de $63 \mathrm{mcg}, 94 \mathrm{mcg}$ y $125 \mathrm{mcg}$, siendo la dosificación una vez cada dos o cuatro semanas.

La eficacia, seguridad y tolerabilidad de peginterferon beta1a se han evaluado en un estudio piloto, multicéntrico, randomizado, doble ciego, paralelo y controlado con placebo de dos años de duración que ha incluido 1516 pacientes. La administración de peginterfeton beta- 1 a cada dos semanas ha reducido la tasa anual de recaídas por un $36 \%(\mathrm{p}=0.0007)$ comparado con placebo, mientras la dosificación cada cuatro semanas la ha reducido por un $28 \%$ ( $p=0.0114)$. Las RAM más comunes fueron eritema en el sitio de inyección, síntomas gripales, fiebre, cefalea, mialgias, escalofríos, astenia y artralgias.

El CHMP ha concedido la autorización de comercialización Plegridy $^{\circledR}$ el 22 de mayo de 2014.

\section{SILTUXIMAB (SYLVANT $\left.{ }^{\circledR}\right)^{17,18}$}

Siltuximab es un anticuerpo monoclonal quimérico que se une y neutraliza los receptores de la interleucina 6 (IL-6) humana con alta afinidad. La IL-6 es una citocina pleiotrópica proinflamatoria producida por diversos tipos celulares, incluyendo las células T y B, monocitos y fibroblastos, además de células malignas. Existen datos de que su producción excesiva desempeña un papel esencial en las manifestaciones sistémicas de los pacientes con la enfermedad de Castleman. Se trata de una patología derivada de la proliferación linfoide anormal y atípica, de causa desconocida, morfológica y clínicamente heterogénea, que se asocia riesgo de linfoma maligno. La forma multicéntrica de esta enfermedad hasta ahora no tenía tratamiento eficaz.

Siltuximab es el primer fármaco con la indicación específica en el tratamiento de la enfermedad de Castleman multicéntrica en pacientes adultos que son negativos para el virus de la inmunodeficiencia humana (VIH) y para el virus herpes humano 8 (VHH-8). El fármaco se presenta en forma de polvo para solución para perfusión y la dosis recomendada es de $11 \mathrm{mg} / \mathrm{kg} / \mathrm{h}$, cada 3 semanas hasta el fracaso del tratamiento. $\mathrm{Su}$ eficacia y seguridad se han evaluado en un estudio fase II controlado con placebo, multinacional, doble ciego, que ha incluido 79 enfermos. La variable principal del estudio era la respuesta tumoral duradera, la cual comprendió una respues- ta parcial o completa de un mínimo de 18 semanas de duración. Se observó una diferencia estadísticamente significativa en cuanto a la variable principal en el brazo de siltuximab comparado con el brazo placebo (34\% vs $0 \%$, respectivamente; IC del 95\%: 11,1; 54,8; p = 0,0012). Las RAM observadas con más frecuencia fueron prurito y exantema maculopapular, infecciones del tracto respiratorio superior, aumento de peso e hiperuricemia.

Fue designado como medicamento huérfano el 30 de noviembre de 2007 .

\section{SIMEPREVIR (OLYSIO $\left.{ }^{\circledR}\right)^{19-22}$}

Simeprevir inhibe la actividad proteolítica de la serina proteasa NS3/4A del VHC, que es esencial para la replicación del virus. Es activo frente las proteasas del VHC recombinante de genotipo la y $1 \mathrm{~b}$, no se ha estudiado su eficacia en pacientes con VHC genotipos 2, 3, 5 ni 6. La dosis recomendada es una cápsula de $150 \mathrm{mg}$ una vez al día durante 12 semanas.

La indicación aprobada es el tratamiento de la infección crónica por el VHC en adultos junto a interferón pegilado y ribavirina (a las dosis convencionales utilizadas en biterapia o terapia triple). Tras cumplir 12 semanas del tratamiento, deberá seguirse una terapia doble con únicamente interferón pegilado y ribavirina durante 12 o 24 semanas. Simeprevir se metaboliza por el citocromo P450 3A4, por lo que pueden tener lugar varias interacciones con otros medicamentos que se metabolicen por la misma vía.

$\mathrm{Su}$ eficacia se ha evaluado en dos estudios fase III, multicéntricos, aleatorizados de dos brazos, doble ciegos y controlados con placebo. Un total de 391 personas participaron en los estudios, las cuales fueron distribuidas de forma aleatoria para recibir 12 semanas de tratamiento con simeprevir (150 mg diarios) o placebo, en ambos casos junto con interferón pegilado y ribavirina. Mientras que en el brazo de placebo solo el 50\% de participantes alcanzaron la respuesta virológica sostenida a las 12 semanas (RVS12), en el grupo de simeprevir el porcentaje fue del $81 \%$. Dentro del grupo de simeprevir, el porcentaje de RVS12 fue máximo en el subgrupo con el genotipo favorable CC de la interleuquina 28B (IL28B), llegando al 96\%. Cabe destacar que el fármaco también se mostró seguro y eficaz en un estudio fase III, abierto, que incluyó 106 pacientes coinfectados con VIH. Las RAM observadas con más frecuencia fueron: náuseas, rash, prurito, disnea, aumento de bilirrubina en sangre y reacciones de fotosensibilidad.

La Comisión Europea ha concedido la autorización de comercialización el 20 de marzo de 2014.

\section{SIMOCTOCOG ALFA $\left(N U W I Q^{\circledR}\right)^{3}$}

Es el factor VIII recombinante que disminuye temporalmente la tendencia a sufrir hemorragias, al incrementar el nivel total de este factor en plasma. Se presenta en forma de polvo y disolvente, para preparar una solución inyectable. Está indicado en el tratamiento y profilaxis de hemorragias en niños y adultos con hemofilia A. 
$\mathrm{Su}$ eficacia en la prevención y tratamiento de hemorragias en cirugía se ha evaluado en 3 ensayos pivotales: en el primer estudio, que incluyó 22 sujetos adolescentes y adultos, el 94.4\% de los episodios de sangrado se controlaron con tratamiento, siendo necesario una sola infusión en la mayoría de los casos. El segundo estudio (32 pacientes adultos), evaluó la eficacia en la profilaxis de eventos hemorrágicos y en el tratamiento de sangrado intercurrente; la tasa media de hemorragias por paciente durante el periodo de tratamiento profiláctico fue de $0.188 / \mathrm{mes}$. En el último estudio (59 pacientes pediátricos), la tasa media de todos los sangrados en profilaxis fue de $0.338 / \mathrm{mes}$, tratándose $81.3 \%$ de las hemorragias con una o dos infusiones. La inmunogenicidad se evaluó en los ensayos clínicos en 135 pacientes previamente tratados con hemofilia A grave, no desarrollando ningún paciente inhibidores del simoctocog alfa.

La Comisión Europea ha concedido la autorización de comercialización en mayo de 2014.

\section{TACROLIMUS (ENVARSUS $\left.{ }^{\circledR}\right)^{23-25}$}

Se trata de tacrolimus de liberación retardada, análogo de Advagraf $^{\circledR}$. Tacrolimus suprime la activación de células $\mathrm{T}$ y la proliferación de células $\mathrm{B}$ dependiente de células T auxiliares, así como la formación de varias linfoquinas responsables del rechazo al trasplante.

Se presenta en comprimidos de liberación retardada de 0.75 mg, $1 \mathrm{mg}$ y $4 \mathrm{mg}$ que se toman una vez al día. Está indicado en adultos para la profilaxis del rechazo del trasplante en receptores de aloinjertos renales o hepáticos, y en el tratamiento del rechazo de aloinjertos resistente a los tratamientos con otros medicamentos inmunosupresores.

El ensayo clínico más importante es un estudio de no inferioridad, fase III, aleatorizado, doble ciego que ha incluido 543 pacientes con trasplante renal "de novo", comparando su eficacia y seguridad frente al tratamiento standard, tacrolimus dos veces al día $\left(\right.$ Prograf $\left.^{\circledR}\right)$. La variable principal era "fallo del tratamiento", una variable compuesta de tres criterios: rechazo de trasplante confirmado por biopsia, recidiva renal y muerte, que se evaluó tras 12 meses del tratamiento. Los resultados mostraron que no hubo diferencias clínicas significativas entre el grupo tratado con Envarsus ${ }^{\circledR}$ y el tratado con tacrolimus de liberación inmediata (18.3\% vs 19.6\%, respectivamente). Las RAM más comunes fueron diarrea, infecciones del tracto urinario, aumento de creatinina en sangre y náuseas.

La Comisión Europea ha concedido la autorización de comercialización en mayo de 2014.

\section{TRAMETINIB (MEKINIST $\left.{ }^{\circledR}\right)^{26,27}$}

Se trata de un inhibidor reversible de las proteínas MEK1 y MEK2, enzimas con actividad quinasa que forman parte de la ruta de señalización mitogénica responsable del control de la proliferación, diferenciación y supervivencia celular en condiciones fisiológicas. Los fallos acaecidos en la regulación de dicha ruta, contribuyen significativamente a la transformación celular y están involucrados en la progresión tumoral. La activación de estas proteínas se produce tras su fosforilacion por parte de dos serina-treonina cinasas, Ras y Raf, cuyas mutaciones han sido relacionadas con varias malignidades. Inhibiendo las proteínas MEK1 y MEK2, trametinib bloquea la transmisión de la señal mitogénica hasta el núcleo en las células de melanoma con BRAF mutado, deteniendo el crecimiento celular in vivo e in vitro.

Trametinib está indicado en monoterapia para el tratamiento de pacientes adultos con melanoma no resecable o metastásico con mutación V600 del gen BRAF. Se presenta en comprimidos de $0,5,1$ y $2 \mathrm{mg}$. Dosis recomendada es de $2 \mathrm{mg}$ al día, hasta la progresión de la enfermedad o desarrollo de toxicidad.

La eficacia y la seguridad se han evaluado en dos ensayos clínicos con pacientes con melanoma metastático o no resecable, positivo para las mutaciones BRAF V600E o V600K. El primer ensayo era internacional, multicéntrico, abierto, aleatorizado, controlado con el tratamiento activo (dacarbazina o paclitaxel) y ha incluido 322 pacientes. El segundo ensayo ha incluido 162 pacientes y ha comparado la eficacia y la seguridad de 2 dosis diferentes de trametinib combinadas con dabrafenib, frente dabrafenib solo. Los ensayos clínicos han mostrado que trametinib mejora la supervivencia global y la supervivencia libre de progresión en pacientes con melanoma con mutación V600 del gen BRAF, tanto en comparación con la quimioterapia convencional como con dabrafenib solo. Las RAM observadas con más frecuencia fueron: erupción cutánea, diarrea, fatiga, edema periférico, náuseas y dermatitis acneiforme.

\section{VEDOLIZUMAB (ENTYVIO $\left.{ }^{\circledR}\right)^{1,29,30}$}

Es un anticuerpo monoclonal inmunoglobulina humanizada G1 (IgG1) dirigido contra la integrina $\alpha 4 \beta 7$ de linfocitos humanos. La integrina $\alpha 4 \beta 7$ se expresa en la superficie de una subpoblación de linfocitos $\mathrm{T}$ de memoria que, mediante la unión con la molécula MAdCAM-1 (Mucosal addressin Cell Adhesion molecule-1) del endotelio vascular intestinal, causan la inflamación característica de la colitis ulcerosa y de la enfermedad de Crohn. Actuando como antagonista selectivo de la integrina $\alpha 4 \beta 7$, vedolizumab impide adhesión de los linfocitos al endotelio intestinal, disminuyendo así su acumulación y producción de citoquinas responsables de la inflamación.

Se presenta en forma de polvo para solución para infusión. Esta indicado en tratamiento de colitis ulcerosa activa moderada a grave y la enfermedad de Crohn activa moderada a grave en los pacientes adultos que han tenido una respuesta inadecuada, no han respondido o son intolerantes al tratamiento convencional o a un antagonista del factor de necrosis tumoral alfa (TNF $\alpha)$.

Se llevaron a cabo dos estudios aleatorizados con vedolizumab doble ciegos y controlados con placebo en pacientes con enfermedad activa. Los pacientes que tuvieron una respuesta a vedolizumab en la semana 6 fueron asignados aleatoriamente para continuar recibiendo vedolizumab cada 4 ó cada 8 semanas, o cambiar a placebo durante 52 semanas. La respuesta se definió como una reducción en la puntuación de la Clínica Mayo (rango 0 a 12, con las puntuaciones más altas indicando la enfermedad más activa) de por lo menos 3 puntos y una disminución de al menos el 30\% del valor basal. En la sexta semana del estudio un 


\section{Evaluación positiva de medicamentos: marzo, abril y mayo 2014}

$47,1 \%$ de los pacientes del grupo vedolizumab respondieron al tratamiento, frente a un $25,5 \%$ del grupo placebo. En la semana 52 alcanzaron la remisión clínica el 44,8\% de los pacientes que siguieron recibiendo vedolizumab cada 4 semanas y el $41,8 \%$ de los pacientes que siguieron recibiendo vedolizumab cada 8 semanas y, vs 15,9\% de los pacientes que cambiaron a placebo.

El CMUH de la Agencia Europea de Medicamentos (EMA) emitió una opinión positiva, recomendando la concesión de una autorización de comercialización de vedolizumab el 20 de marzo de 2014.

\section{VINTAFOLIDA ( VYNFINIT $\left.{ }^{\circledR}\right)^{1,31,32}$}

Con el fin de satisfacer la demanda de folato, ciertos tipos de tumores - como el cáncer de ovario- expresan altas concentraciones de receptores del folato en su superficie. Vintafolida es un combinado de ácido fólico y del agente citotóxico desacetilvinblastina hidrazida (DAVLBH), un alcaloide de la vinca derivado de vinblastina que se administra por vía intravenosa. El ácido fólico permite que se libere DAVLBH preferentemente en células cancerosas que expresan el receptor de folato. Una vez liberado dentro de las células, DAVLBH actúa inhibiendo el ensamblaje de los microtúbulos y deteniendo a las células en mitosis.

Esta indicado en combinación con doxorubicina liposomal pegilada (DLP) para el tratamiento de pacientes adultas con cáncer de ovario resistente a platino que expresen el receptor de folato en las lesiones a tratar. Antes de iniciar el tratamiento con vintafolida se debe evaluar el estado del receptor de folato mediante el uso de etafolida, utilizando SPECT, en combinación con CT o RM.

La autorizacion de vintafolida se basa en los resultados de un estudio aleatorio de fase IIb llevado a cabo en pacientes con cáncer de ovario platino resistente a los que se ha tratado con vintafolida tras la administración de etarfolida y la realización de SPECT. Vintafolida en combinación con DLP frente a DLP más placebo se está estudiando actualmente en un ensayo clínico en fase III, aleatorio y doble ciego en pacientes con cáncer de ovario platino resistente que sobreexpresa receptores de folato. El criterio de valoración principal del ensayo es la supervivencia libre de progresión de la enfermedad determinada por los criterios RECIST (criterios de evaluación de la respuesta en pacientes con tumores sólidos). Las RAM observadas con mayor frecuencia fueron: fatiga, estomatitis, neutropenia, anemia, náuseas y neuropatía sensorial periférica.

El CHMP ha recomendado de manera condicional la autorización de comercialización de vintafolida, en espera de los resultados del estudio PROCEED. Vynfinit ${ }^{\circledR}$ fue designado como medicamento huérfano el 9 de febrero de 2012.

\section{BIBLIOGRAFIA}

1. Agencia Española de Medicamentos y Productos Sanitarios. Informe mensual sobre Medicamentos de Uso Humano y Productos Sanitarios Marzo 2014. Disponible en: www.aemps.gob.es/informa/boletinMensual/.../marzo/ boletin-marzo.htm
2. Committee for Medicinal Products for Human Use (CHMP). Folic acid. Disponible en: http://www.ema.europa.eu/docs/en_GB/document_library/Summary_of_opinion_-_Initial_authorisation/human/002773/WC500163557.pdf

3. Agencia Española de Medicamentos y Productos Sanitarios. Informe mensual sobre Medicamentos de Uso Humano y Productos Sanitarios Mayo 2014. Disponible en: www.aemps.gob.es/informa/boletinMensual/.../mayo/ boletin-mayo.htm

4. McDonald CM et al: "The 6-minute walk test and other endpoints in Duchenne muscular dystrophy: longitudinal natural history observations over 48 weeks from a multicenter study." Muscle Nerve. 2013 Sep;48(3):343-56. Disponible en: http://www.ncbi.nlm.nih.gov/pmc/articles/PMC3824082/

5. Simbrinza. Disponible en: http://ecatalog.alcon.com/pi/simbrinza_us_en.pdf

6. Autores no listados. "Brinzolamide/brimonidine (Simbrinza) for glaucoma." Med Lett Drugs Ther. 2013 Jul 22;55(1421):57-8. Disponible en: http://www. ncbi.nlm.nih.gov/pubmed/23863917

7. Jardiance.. Ficha técnica de la Agencia Europea de Medicamentos. Disponible en: http://ec.europa.eu/health/documents/community-register/2014/20140522128562/anx_128562_es.pdf

8. Ridderstråle $\mathrm{M}$. et al: "Rationale, design and baseline characteristics of a 4-year (208-week) phase III trial of empagliflozin, an SGLT2 inhibitor, versus glimepiride as add-on to metformin in patients with type 2 diabetes mellitus with insufficient glycemic control." Cardiovasc Diabetol. 2013 Sep 5;12:129. Disponible en: http://www.ncbi.nlm.nih.gov/pmc/articles/ PMC3844307/

9. Rosenstock J. et al:" Efficacy and safety of empagliflozin, a sodium glucose cotransporter 2 (SGLT2) inhibitor, as add-on to metformin in type 2 diabetes with mild hyperglycaemia." Diabetes Obes Metab. 2013 Dec;15(12):1154-60. Disponible en: http://www.ncbi.nlm.nih.gov/pubmed/23906374

10. Phillip H. Kuo: "Companion Imaging Diagnostics for Targeted Therapies". Radiology Today14:32. Disponible en: http://www.radiologytoday.net/archive/rt0213p12.shtml\#sthash.YGxGSHyc.dpuf

11. Committee for Medicinal Products for Human Use (CHMP).Etarfolatide. Disponible en: http://www.ema.europa.eu/docs/en_GB/document_library/Summary_of_opinion_-_Initial_authorisation/human/002570/ WC500163555.pdf

12. PRECEDENT: a randomized phase II trial comparing vintafolide (EC145) and pegylated liposomal doxorubicin (PLD) in combination versus PLD alone in patients with platinum-resistant ovarian cancer. Disponible en: http:// www.clinicaltrials.gov/ct2/show/NCT01170650?term=Endocyte\&rank=1

13. Committee for Medicinal Products for Human Use (CHMP). Obinutuzumab. Disponible en: http://www.ema.europa.eu/docs/en_GB/document_library/Press_release/2014/05/WC500167542.pdf

14. Goede V. et al: "Obinutuzumab plus chlorambucil in patients with CLL and coexisting conditions.” N Engl J Med. 2014 Mar 20;370(12):1101-10.

15. Committee for Medicinal Products for Human Use (CHMP). Peginterferon beta-1. Disponible en: http://www.ema.europa.eu/docs/en_GB/document_library/Summary_of_opinion_-_Initial_authorisation/human/002827/ WC500167334.pdf

16. Plegridy. Disponible en: www.biogenidec.com/.../Download.aspx?file

17. Silvant. Ficha técnica de la Agencia Europea de Medicamentos. Disponible en: http://ec.europa.eu/health/documents/community-register/2014/20140522128587/anx_128587_es.pdf

18. Silvant. Disponible en: http://www.sylvant.com/sites/default/files/pdf/ SYLVANT(siltuximab).pdf

19. Olysio. Ficha técnica de la Agencia Europea de Medicamentos. Disponible en: http://www.ema.europa.eu/docs/es_ES/document_library/EPAR_-_Product_Information/human/002777/WC500167867.pdf

20. Jacobson I, Dore GJ, Foster GR, et al. :"Simeprevir (TMC435) with peginterferon/ribavirin for treatment of chronic HCV genotype 1 infection in treatment-naïve patients: results from QUEST-1 a phase III trial". Lancet. 2014 Jun 3. pii: S0140-6736(14)60494-3. Disponible en: http://www.thelancet. com/journals/lancet/article/PIIS0140-6736(14)60494-3/fulltext

21. Manns M, Marcellin P, Poordad F, et al. "Simeprevir (TMC435) with peginterferon/ribavirin for treatment of chronic HCV genotype 1 infection in treatment-naive patients: results from QUEST-2 a phase III trial". Lancet. 2014 Jun 3. pii: S0140-6736(14)60538-9. Disponible en: http://www.thelancet. com/journals/lancet/article/PIIS0140-6736(14)60538-9/fulltext

22. Dieterich D, Rockstroh J, Orkin C, et al. "Simeprevir (TMC435) with Peginterferon/Ribavirin in patients Co-infected with HCV genotype-1 and HIV- 
1: Primary analysis of the C212 Study.”. Disponible en: http://www.natap. org/2013/EACS/EACS_15.htm

23. Committee for Medicinal Products for Human Use (CHMP). Tacrolimus. Disponible en: http://www.ema.europa.eu/ema/index.jsp?curl=pages/medicines/human/medicines/002655/smops/Positive/human_smop_000689. jsp\&mid=WC0b01ac058001d127

24. Envarsus. Disponible en: http://www.veloxis.com/envarsus.cfm

25. Tsuchiya et al: "Comparison of pharmacokinetics and pathology for lowdose tacrolimus once-daily and twice-daily in living kidney transplantation: prospective trial in once-daily versus twice-daily tacrolimus. "Transplantation. 2013 Jul 27;96(2):198-204. Disponible en: http://www.ncbi.nlm.nih.gov/ pubmed/23792649

26. Mekinist. Ficha técnica de la Agencia Europea de Medicamentos. Disponible en: http://www.accessdata.fda.gov/drugsatfda_docs/ label/2013/204114s000lbl.pdf

27. Siegfried Ussar et al: "MEK1 and MEK2, Different Regulators of the G1/S Transition.” Disponible en: http://www.jbc.org/content/279/42/43861.long
28. Soler D et al. "The binding specificity and selective antagonism of vedolizumab, an anti- $\alpha 4 \beta 7$ integrin therapeutic antibody in development for inflammatory bowel diseases. J Pharmacol Exp Ther. 2009;330(3):864-875. Disponible en: http://jpet.aspetjournals.org/content/330/3/864.full.pdf+html.

29. Gledhill T et al." New and emerging treatments for ulcerative colitis: a focus on vedolizumab.” Biologics: targets and therapy 2013;7:123-130. Disponible en: http://www.ncbi.nlm.nih.gov/pmc/articles/PMC3666591/

30. Committee for Medicinal Products for Human Use (CHMP). Vedolizumab. Disponible en: http://www.ema.europa.eu/docs/en_GB/document_library/Summary_of_opinion_-_Initial_authorisation/human/002782/ WC500163492.pdf

31. Parker N. at al." Folate receptor expression in carcinomas and normal tissues determined by a quantitative radioligand binding assay.” Anal Biochem. 2005 Mar 15;338(2):284-93. Disponible en: http://www.sciencedirect.com/science/ article/pii/S0003269705000060

32. Vintafolida. PROCEED clinical trial. Disponible en: http://www.clinicaltrials.gov/ct2/show/NCT01170650?term=Endocyte\&rank=1 